\title{
Research Article \\ Solving the Tractor and Semi-Trailer Routing Problem Based on a Heuristic Approach
}

\author{
Hongqi Li, ${ }^{1}$ Yue Lu, $^{1}$ Jun Zhang, $^{2}$ and Tianyi Wang ${ }^{1}$ \\ ${ }^{1}$ School of Transportation Science and Engineering, BeiHang University, No. 37 Xueyuan Road, \\ Haidian District, Beijing 100191, China \\ ${ }^{2}$ School of Economics and Management, BeiHang University, No. 37 Xueyuan Road, Haidian District, \\ Beijing 100191, China
}

Correspondence should be addressed to Hongqi Li, lihongqi@buaa.edu.cn

Received 27 February 2012; Accepted 27 May 2012

Academic Editor: Jianming Shi

Copyright (C) 2012 Hongqi Li et al. This is an open access article distributed under the Creative Commons Attribution License, which permits unrestricted use, distribution, and reproduction in any medium, provided the original work is properly cited.

\begin{abstract}
We study the tractor and semi-trailer routing problem (TSRP), a variant of the vehicle routing problem (VRP). In the TSRP model for this paper, vehicles are dispatched on a trailer-flow network where there is only one main depot, and all tractors originate and terminate in the main depot. Two types of decisions are involved: the number of tractors and the route of each tractor. Heuristic algorithms have seen widespread application to various extensions of the VRP. However, this approach has not been applied to the TSRP. We propose a heuristic algorithm to solve the TSRP. The proposed heuristic algorithm first constructs the initial route set by the limitation of a driver's on-duty time. The candidate routes in the initial set are then filtered by a two-phase approach. The computational study shows that our algorithm is feasible for the TSRP. Moreover, the algorithm takes relatively little time to obtain satisfactory solutions. The results suggest that our heuristic algorithm is competitive in solving the TSRP.
\end{abstract}

\section{Introduction}

In this paper, we consider the tractor and semi-trailer routing problem (TSRP), a variant of the vehicle routing problem (VRP). The VRP is one of the most significant problems in the fields of transportation, distribution, and logistics. The basic VRP consists of some geographically dispersed customers, each requiring a certain weight of goods to be delivered (or picked up). A fleet of identical vehicles dispatched from a depot is used to deliver the goods, and the vehicles must terminate at the depot. Each vehicle can carry a limited weight and only one vehicle is allowed to visit each customer. It is assumed that some parameters (e.g., customer demands and travel times) are known with certainty. The solution of the problem consists 
of finding a set of routes that satisfy the freight demand at minimal total cost. In practice, additional operational requirements and restrictions, as in the case of the truck and trailer routing problem (TTRP), may be imposed on the VRP [1]. The TTRP was first studied by Semet and Taillard et al. [2] and Gerdessen [3] in the 1990s, and it was subsequently studied by Chao [4], Scheuerer [5], and others. In the TTRP, the use of trailers (a commonly neglected feature in the VRP) is considered. Some customers can be served by a combination vehicle (i.e., a truck pulling a trailer, as in type II in Figure 1), while other customers can only be served by a truck (as type I in Figure 1) due to some limitations such as government regulations, limited maneuvering space at customer sites, road conditions, and so forth. These constraints exist in many practical situations [1].

The VRP and its various extensions have long been one of the most studied combinatorial optimization problems due to the problem's complexity and extensive applications in practice [6-10]. The truck and trailer combination is employed widely by enterprises around the world, but there additional features introduced by trailers that have attracted some research. A number of studies have concentrated on applications of the TTRP. For instance, Semet and Taillard et al. [2] and Caramia and Guerriero et al. [11] gave some real-world TTRP applications in collection and delivery operations in rural areas or crowded cities with accessibility constraints. Theoretically, being an extension of the VRP, the TTRP is NP-Hard. The TTRP is computationally more difficult to solve than the VRP [1]. Because the VRP is usually tackled by heuristic methods $[6-9,12-15]$, it is feasible to develop heuristic approaches for the TTRP.

Gerdessen [3] extended the VRP to the vehicle routing problem with trailers and investigated the optimal deployment of a fleet of truck-trailer combinations by a construction and improvement heuristic. Scheuerer [5] proposed construction heuristics (called T-Cluster and T-Sweep) along with a tabu search algorithm for the TTRP. Tan et al. [16] proposed a hybrid multiobjective evolutionary algorithm featuring specialized genetic operators, variable-length representation; and local search heuristics to solve the TTRP. Lin et al. [1] proposed a simulated annealing (SA) heuristic for the TTRP and suggested that SA is competitive with tabu search (TS) for solving the TTRP. Villegas et al. [17] solved the TTRP by using a hybrid metaheuristic based on a greedy randomized adaptive search procedure (GRASP), variable neighborhood search (VNS), and path relinking (PR).

Villegas et al. [18] proposed two metaheuristics based on GRASP, VND, and evolutionary local search (ELS) to solve the single truck and trailer routing problem with satellite depots (STTRPSD). Considering the number of available trucks and trailers to be limited in the TTRP, Lin et al. [19] relaxed the fleet size constraint and developed a SA heuristic for solving the relaxed truck and trailer routing problem (RTTRP). Lin et al. [20] proposed a SA heuristic for solving the truck and trailer routing problem with time windows (TTRPTW).

Research to date has considered most types of road vehicles, especially trucks and truck and trailer combinations. However, there has been little research on the types of tractor and semi-trailer combinations. Hall and Sabnani et al. [21] studied routes that consisted of two or more segments and two or more stops in the tour for a tractor. At each stop, the tractor could drop off one or more trailers and pick up one or more trailers. Control rules based on predicted route productivity were developed to determine when to release a tractor. Derigs et al. [22] presented two approaches to solve the vehicle routing problem with multiple uses of tractors and trailers. The primary objective was to minimize the number of required tractors. Cheng et al. [23] proposed a model for a steel plant to find the tractor and semi-trailer equipment and running routes for the purpose of minimizing transport distance. Liang [24] 


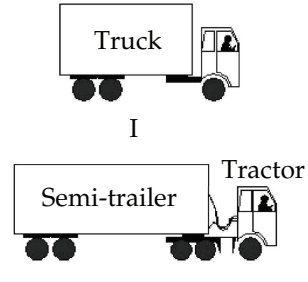

III

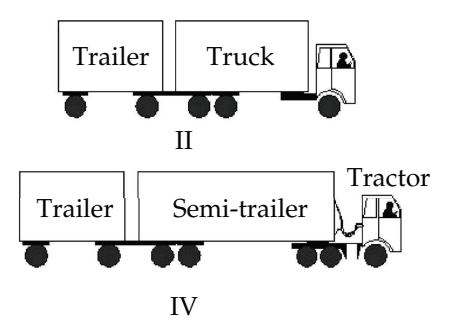

IV

Figure 1: The basic types of vehicles. Note: In practice, many vehicle types are used in road freight transportation. This figure only lists four basic types. A large number of other types can be derived from the four basic types by the number of axles, tires and the combination style. Enterprises in most of the countries in the world employ various types.

established a dispatching model of tractors and semi-trailers in a large steel plant and used a tabu search algorithm to find the optimal driving path and the cycle program.

We aim to propose a heuristic for the TSRP. This aim is based on the practical knowledge that tractor and semi-trailer combinations are popular in some countries, particularly China. The remainder of this paper is organized as follows. Section 2 compares the TTRP and the TSRP and defines the TSRP. Section 3 proposes a heuristic algorithm to solve the TSRP. Section 4 employs the heuristic algorithm to solve some experimental networks of the TSR. Section 5 draws conclusions and gives future research directions.

\section{Problem Definition}

\subsection{The TTRP and the TSRP}

Although there is little literature devoted to the definition and solution of the TSRP in the fields of transportation or logistics, plenty of research has been done on the TTRP, providing important references for the TSRP. In the TTRP, a heterogeneous fleet composed of $m_{\mathrm{tu}}$ trucks and $m_{\mathrm{tr}}$ trailers $\left(m_{\mathrm{tu}}>m_{\mathrm{tr}}\right)$ serves a set of customers from a main depot. Each customer has a certain demand, and the distances between any two points (including customers and depots) are known. The capacities of the trucks and trailers are determinate. Some customers must be served only by a truck, while other customers can be served either by a truck or by a combination vehicle. The objective of the TTRP is to find a set of routes with minimum total distance or cost so that each customer is visited in a route performed by a compatible vehicle, the total demand of the customers visited on a route does not exceed the capacity of the allocated vehicle, and the numbers of required trucks and trailers are not greater than $m_{\mathrm{tu}}$ and $m_{\mathrm{tr}}$, respectively $[1,17]$. There are three types of routes in a TTRP solution, as illustrated in Figure 2: (1) a pure truck route traveled by a single truck; (2) a pure vehicle route without any subtour traveled by a combination vehicle; (3) a combination vehicle route consisting of a main tour traveled by a combination vehicle and at least one subtour traveled by the truck alone.

The vehicle types in the TSRP are different from those in the TTRP. The TTRP focuses on trucks and trailers, both of which can carry cargo. The TSRP involves $m_{\mathrm{ta}}$ tractors and $m_{\text {st }}$ semi-trailers $\left(m_{\mathrm{ta}}<m_{\mathrm{st}}\right)$. Although a tractor cannot carry cargo, it has more flexible dispatching options, and it can pull different semi-trailers on various segments of its route by the pick-up and drop-off operation at depots. 


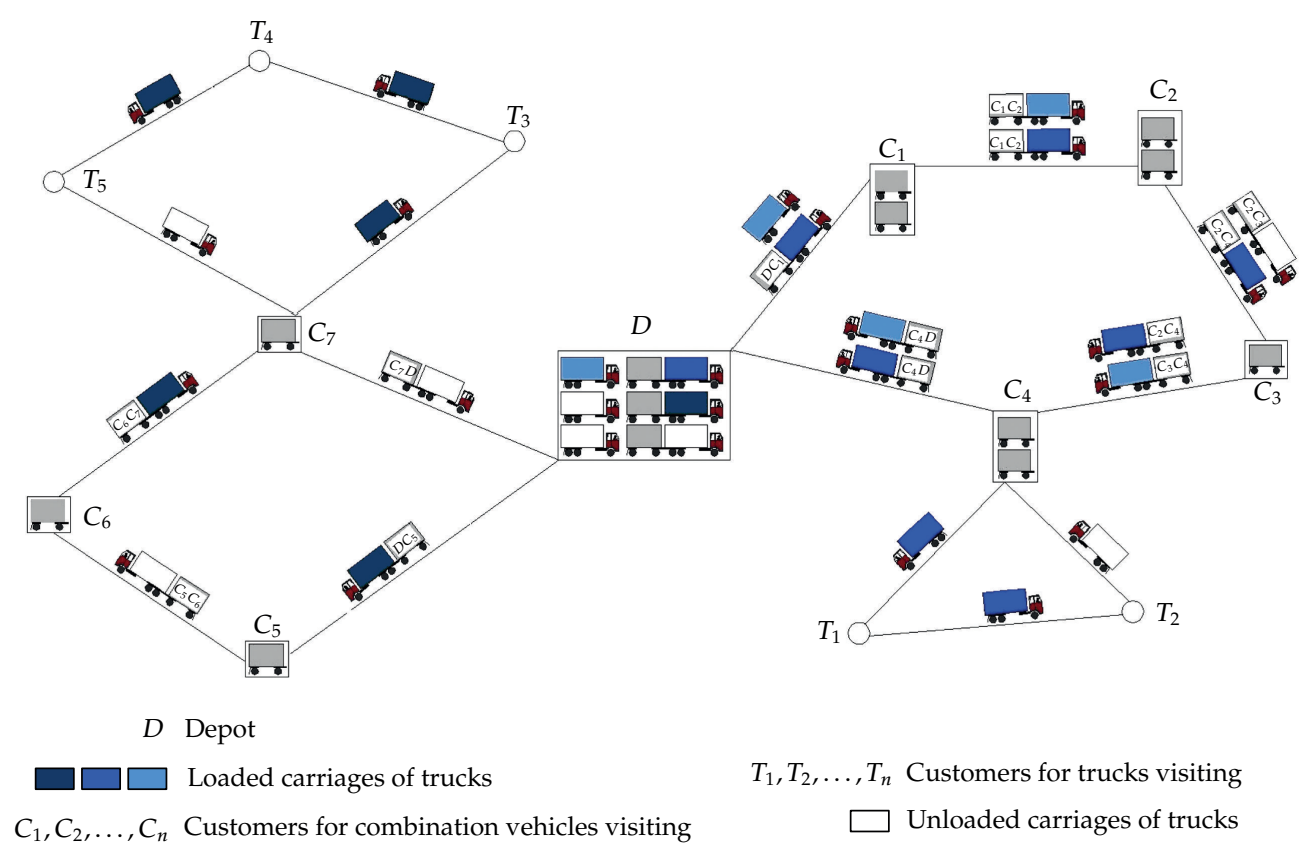

Figure 2: Different types of vehicle routes in the TTRP.

The TSRP can be formally defined on a directed graph $G=(V, A)$, where $V=$ $\{0,1,2, \ldots, n\}$ is the set of vertices and $A=\{(i, j): i, j \in V\}$ is the set of arcs. Each arc $(i, j)$ is generally associated with a transportation distance decided by road infrastructure. The freight flow from $i$ to $j$ is regarded as certain weight of $\operatorname{arc}(i, j)$. Vertex $0, \ldots, v(v<n)$ represents the main depots, in which many tractors and semi-trailers park. Some loaded semi-trailers wait for visiting customers, and other unloaded semi-trailers wait for visiting or maintenance. The remaining vertices $\left(s_{i}\right)$ in $V$ (i.e., $\left.V \backslash\{0, \ldots, v\}\right)$ correspond to customers who have $m(m \geq 1)$ loaded semi-trailers waiting to visit $l(1 \leq l \leq n$ and $l \leq m)$ orientations at the beginning of the simulation. Customers may have other unloaded semi-trailers waiting for loading.

There are various tractor-driving modes on graph $G$ during one daily period. For example, (1) tractor $\operatorname{Tr}_{j}$, pulling one loaded semi-trailer, goes from its main depot to a customer in one-day period, and the customer has tractor-parking available; (2) tractor $\operatorname{Tr}_{j}$, pulling one loaded semi-trailer, goes from its main depot to $c_{1}$. After the dropping and pulling operations at $c_{1}$, the tractor goes on to another customer, $c_{2}$. The tractor $\operatorname{Tr}_{j}$ terminates at a customer who has tractor-parking available. (3) It is similar to the running course listed in (2), but Tractor $\operatorname{Tr}_{j}$ terminates at its main depot. The most basic elements of tractor-driving modes include the following: how many semi-trailers can be pulled synchronously by a tractor, how many vertexes are passed by the tractor, how many times per day the tractor can drop off one or more trailers and pick up one or more trailers, whether a tractor terminates at its original main depot, whether the semi-trailer pulled by a tractor loads cargo, and if a tractor runs alone. In addition, a time window constraint is probably required. 


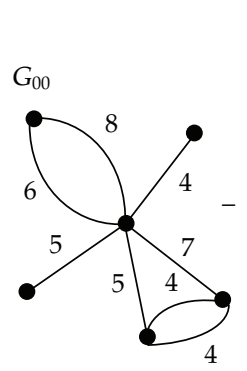

The first selection

$G_{01}$

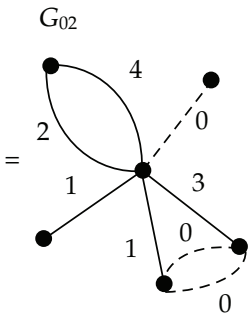

The second selection
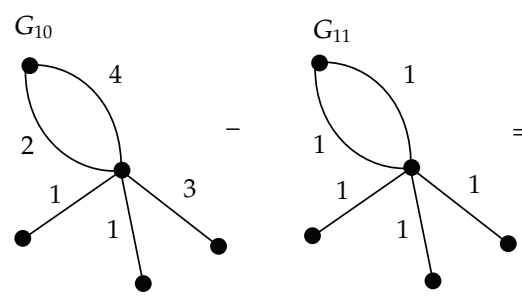

$G_{12}$

The third selection
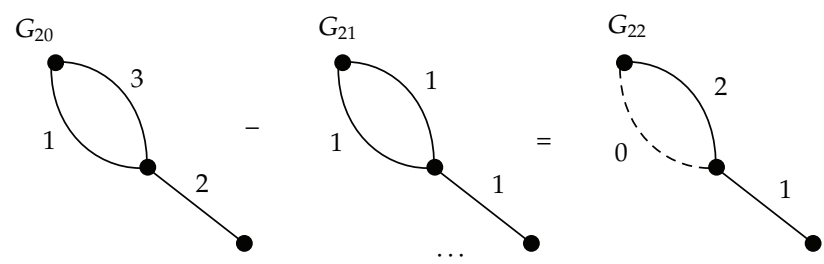

Figure 3: An example of selecting unit-flow networks. Note: Black points denote depots. Lines denote the distribution of freight flows. Numbers near lines denote freight flow volume (unit: one semi-trailer).

\subsection{The TSRP Model}

In practice, there are many depots on the freight transportation network of an enterprise. Depots have different functions and sizes. In the TSRP, we classify these depots into two types: main depots and customer depots. The flow of freight between any two depots is usually uneven. In our method, we abstract the freight transportation network onto a graph (denoted by $G_{00}$ ). Graph $G_{00}$ has one main depot and a number of customer depots where semi-trailers can park. Initially, all tractors are parked in the main depot, and semi-trailers that carry cargos are waiting for transport.

Because the freight flows among various depots are unequal, we select a freight flow network denoted by $G_{01}$. $G_{01}$ is a subset of $G_{00}$ on which the freight flows among various depots are equal. Graph $G_{01}$ is probably a combination of some unit-flow network. On a unitflow network, the freight flow on every arc is one semi-trailer. We denote the subset $G_{00}-G_{01}$ by $G_{02}$. We go on to select another equal flow network (denoted by $G_{11}$ ) from $G_{02}$. After repeating this process several times, the original freight flow network is split into several unit-flow networks (e.g., $G_{01}, G_{11}$, and $G_{21}$ in Figure 3). The study of unit-flow networks is meaningful and important in solving the TSRP. 


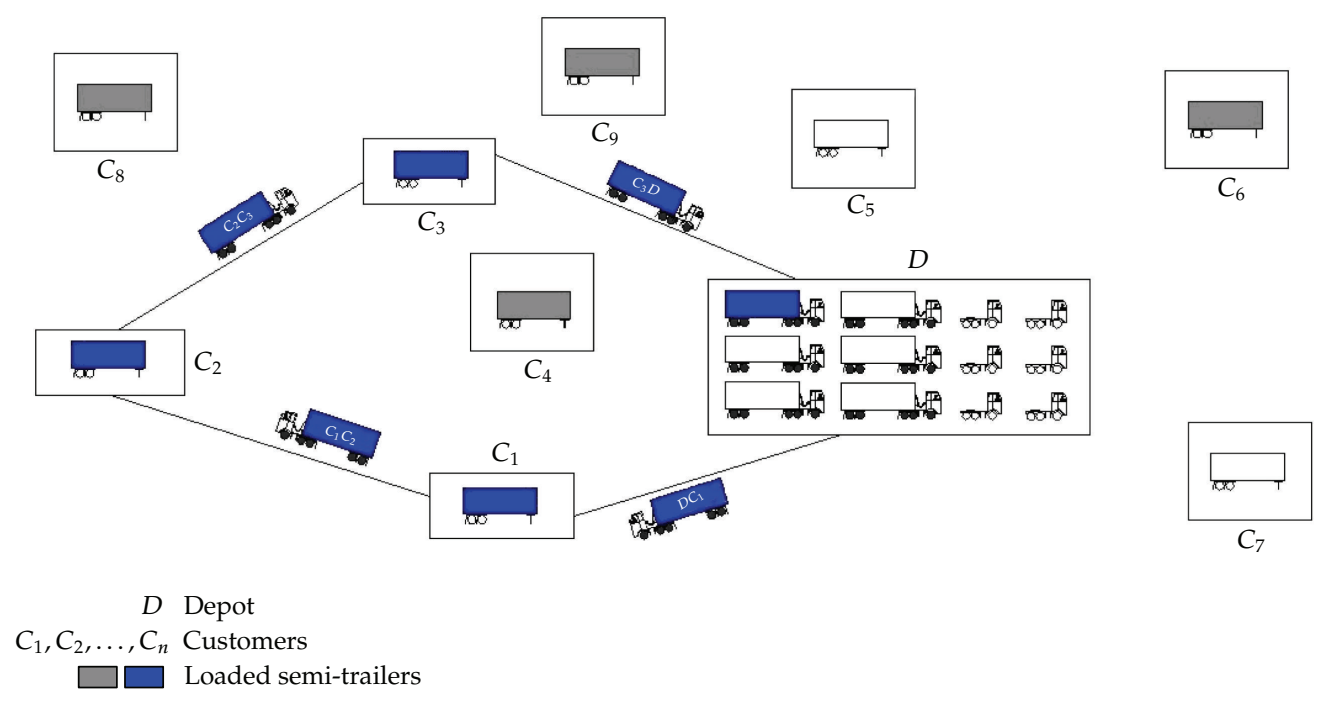

Figure 4: Vehicle routes in the TSRP model.

The TSRP model in this paper uses the unit-flow network. Vertex 0 represents the main depot where some loaded semi-trailers are waiting to be delivered to customers. The vertices in $V \backslash\{0\}$ correspond to customers who have $m(1 \leq m \leq n)$ loaded semi-trailers waiting for going to $m$ customers. The $n$ customers have no parked tractors. The tractor-driving modes must satisfy some constraints, including the following: tractors terminate at the main depot, a tractor can pull one loaded semi-trailer and can also run alone, and the working time of a tractor is decided by its driver team. All tractors or vehicles (a vehicle is one tractor pulling one semi-trailer) originate and terminate at the main depot. Whenever a tractor passes by a customer, the tractor picks up a semi-trailer. Whenever a vehicle passes by a customer, the vehicle drops off its semi-trailer and picks up another one. After one-day period, the number of semi-trailers parked in every customer point is not less than a minimum (Figure 4).

The TSRP model consists of determining the number of tractors to be used and the route of each tractor so that the variable costs and service level are balanced, while each route starts and ends at the main depot. Variable costs are reduced by decreasing the overlap distance of tractors running alone. The service level is based on the percentage of customer demand that is satisfied.

\section{A Heuristic Algorithm for the TSRP}

\subsection{Construct the Initial Solution Set}

Drivers are assigned to ensure flexible running of tractors. A driver's on-duty hours per day are determined by the legal on-duty time and driver dispatching mode. On-duty hours consist of driving hours plus temporary rest time or residence time in depots. The residence time at the main depot (denoted by $H$ ) is for pick-up, drop-off, and some essential maintenance work on vehicles. The temporary rest time at other depots (denoted by $s_{i}$ $(i=1,2, \ldots))$ is for pick-up and/or drop-off semi-trailers. The driving hours restrict tractor running time. 
The elements of the initial solution set are tractor routes. To give the form of a route, we suggest the following procedure. The number of drivers assigned to each tractor is $k$. The on-duty time of each driver is $T$ (hours per person - day). The distance between depots $i$ and $j$ is $d_{i j}$. The depot sequence on each route is denoted by $H-s_{1}-\cdots-s_{f}-H$, which is the form of a route. The same customer is visited only once on a certain tractor's route, and each $s_{i}(i=1,2, \ldots f)$ is unique. The on-duty time $T$ is a constraint on the route. That is,

$$
\rho_{1} \cdot k T \leq \sum_{i} \sum_{j}\left(\frac{d_{i j}}{v}\right)+f \cdot t_{s}+2 \cdot t_{H} \leq \rho_{2} \cdot k T,
$$

where $t_{S}$ and $t_{H}$ are the temporary rest time and the residence time, respectively. $\rho_{1}\left(0<\rho_{1}<\right.$ $1)$ and $\rho_{2}\left(1 \leq \rho_{2} \leq \tau, \tau\right.$ is a limited number) are the lower and upper limits of the utilization ratio of the on-duty time, respectively. $v$ is the average velocity of the tractor.

We suggest the steps below to construct elements of the initial solution set.

Step 1. Transform the distance matrix into a running time matrix. Use $v$ as the parameter in the transformation. Factors affecting $v$ in practice include the tractor condition, the driver's skill, and traffic conditions. We estimate $v$ by enterprise experience.

Step 2. Search the running time matrix. Let $f$ be the sum of customers on a route. If $f$ is very large, there are too many customers on the route to allow too much temporary rest time. Therefore, $f$ has a maximum, and the maximum is certainly less than $\left(k T-2 \cdot t_{H}\right) / t_{s}$. Once $f$ is found, we implement an entire search on the running time matrix to find all routes that satisfy the constraint (3.1).

Step 3. Compare the routes with freight flow demand. Every route in Step 2, which contains many segments, is constructed by the segment running time of all customer pairs. In fact, not all pairs of customers require freight exchange. There are segments on which no freight flows, and tractors run alone on such segments. To save variable costs, the time of tractors running alone is limited. Therefore, we obtain the initial solution set after the elimination of routes based on the freight flow demand and the cost-saving requirement.

\subsection{A 2-Phase Approach to Improve the Initial Solution Set}

\subsubsection{The First Phase}

The more elements (i.e., tractor routes) in the initial solution set, the more choices for freight enterprises. We classify tractor routes into certain types, according to the number of customers on a route. There is one customer passed by in the 1st type, two customers passed by in the 2nd type, and so on. When there are many customers on a route, the tractor can serve more freight demand. When more temporary rest time is consumed at customer points, the effective running hours of the tractor are reduced. Routes of the same type generally have some "overlap arcs" in which only one tractor pulls a semi-trailer, and others run alone. We suggest reducing the total distance of "overlap arcs."

(1) The first step is for the same type. An overlap arc $(i, j)$ where the tractor running time is less than a maximum $\left(t_{i j}\right)_{\max }$ can be accepted. If the tractor needs more time 
than $\left(t_{i j}\right)_{\max }$ on arc $(i, j)$, only one of the routes containing arc $(i, j)$ is permitted to be chosen.

(2) The second step is for different types. A "tractor route-overlap arc" matrix $\left(A_{0}\right.$, with elements $\left.a_{i k}\right)$ is constructed. The rows of matrix $A_{0}$ are serial numbers $\left(\mathrm{sn}_{i}\right)$ of routes and the columns are various overlap arcs. The elements of matrix $A_{0}$ are 1 or 0 . If the element on row $i$ and column $k$ is " 1 ", then the $\mathrm{sn}_{i}$ route has an overlap arc. When $a_{i k}=1$, any elements of the matrix that satisfy $a_{i j}=1$ are considered. The column which contains $a_{i j}$ has a sum $\sum_{i} a_{i j}$. If the route with serial number $\mathrm{sn}_{i}$ is chosen, $\sum_{j} \sum_{i} a_{i j}$ should be at a minimum. Once the $\mathrm{sn}_{i}$ route is chosen, other routes that have the same overlap arcs with the $\mathrm{sn}_{i}$ route are eliminated from $A_{0}$. Consequently, a row in $A_{0}$ changes, and a new matrix $A_{1}$ appears. The operation is repeated until there is no row available in the last "tractor route-overlap arc" matrix.

In the first phase, a transitional solution set that contains such elements as the $\mathrm{sn}_{i}$ routes is constructed by improving the initial solution set.

\subsubsection{The Second Phase}

In the second phase, we propose the "fill-and-cut" approach to attain a satisfactory solution to the TSRP.

Step 1. Construct a zero matrix $O$ (its elements are $o_{i j}$ ) whose rows and columns are depots (i.e., the main depot and customer depots). Because of transportation demand, there are freight flows between two particular depots. Because the segments of tractor routes in the transitional solution set are defined by depots, we add 1 to $o_{i j}$ when there is a route containing arc $(i, j)$. We call such an operation a "fill". By a "fill" operation, we mark all segments of routes in the transitional solution set on matrix $O$. A new matrix $B_{0}$ is thus formed.

Step 2. All route segments in the transitional solution set actually have corresponding elements in matrix $B_{0}$. If a certain percentage (e.g., $80 \sim 100 \%$ ) of all corresponding elements of the $\mathrm{sn}_{i}$ route are greater than 1 , the $\mathrm{sn}_{i}$ route is eliminated. When the $\mathrm{sn}_{i}$ route is eliminated, all of the corresponding elements subtract 1 . We call such an operation a "cut". Repeat the "cut" operation, and a new matrix $B_{1}$ finally forms. The routes corresponding to $B_{1}$ make up the satisfactory solution set.

In some cases, the number of nonzero elements of $B_{1}$ is less than that of the freight flow demands . Therefore, routes corresponding to $B_{1}$ cannot satisfy all transportation demand. In order to satisfy more customers' demands, we can add some routes that contain overlap arcs to increase the market adaptability of the satisfactory solution. However, too many overlap arcs can exist because of uneven freight flows. Therefore, to balance the service level and costs, meeting a certain percentage (e.g., 80\%) of all transportation demand can be the objective.

\section{Computational Study}

We abstract the transportation network on an $\mathrm{N} \times \mathrm{N}$ grid, where the nodes denote the main depot and customer depots. In our computational study, the "RANDOM" function in Matlab, 
Table 1: The tractor running time between two depots (hours).

\begin{tabular}{|c|c|c|c|c|c|c|c|c|c|c|c|c|c|c|}
\hline \multirow{2}{*}{ From } & \multicolumn{14}{|c|}{ To } \\
\hline & $H$ & 1 & 2 & 3 & 4 & 5 & 6 & 7 & 8 & 9 & 10 & 11 & 12 & 13 \\
\hline $\bar{H}$ & 0 & 5.5 & 4.0 & 4.5 & 1.5 & 1.0 & 1.0 & 4.0 & 3.0 & 4.5 & 1.0 & 3.5 & 3.5 & 3.0 \\
\hline 1 & 5.5 & 0 & 1.5 & 1.0 & 4.0 & 4.5 & 4.5 & 2.5 & 2.5 & 3.0 & 5.5 & 4.0 & 5.0 & 6.5 \\
\hline 2 & 4.0 & 1.5 & 0 & 2.5 & 2.5 & 3.0 & 3.0 & 4.0 & 3.0 & 4.5 & 4.0 & 3.5 & 3.5 & 5.0 \\
\hline 3 & 4.5 & 1.0 & 2.5 & 0 & 3.0 & 3.5 & 3.5 & 1.5 & 1.5 & 2.0 & 4.5 & 3.0 & 4.0 & 5.5 \\
\hline 4 & 1.5 & 4.0 & 2.5 & 3.0 & 0 & 0.5 & 0.5 & 4.5 & 3.5 & 5.0 & 1.5 & 4.0 & 4.0 & 3.5 \\
\hline 5 & 1.0 & 4.5 & 3.0 & 3.5 & 0.5 & 0 & 1.0 & 5.0 & 4.0 & 5.5 & 2.0 & 4.5 & 4.5 & 4.0 \\
\hline 6 & 1.0 & 4.5 & 3.0 & 3.5 & 0.5 & 1.0 & 0 & 4.0 & 3.0 & 4.5 & 1.0 & 3.5 & 3.5 & 3.0 \\
\hline 7 & 4.0 & 2.5 & 4.0 & 1.5 & 4.5 & 5.0 & 4.0 & 0 & 1.0 & 0.5 & 4.0 & 2.5 & 3.5 & 5.0 \\
\hline 8 & 3.0 & 2.5 & 3.0 & 1.5 & 3.5 & 4.0 & 3.0 & 1.0 & 0 & 1.5 & 3.0 & 1.5 & 2.5 & 4.0 \\
\hline 9 & 4.5 & 3.0 & 4.5 & 2.0 & 5.0 & 5.5 & 4.5 & 0.5 & 1.5 & 0 & 3.5 & 2.0 & 3.0 & 4.5 \\
\hline 10 & 1.0 & 5.5 & 4.0 & 4.5 & 1.5 & 2.0 & 1.0 & 4.0 & 3.0 & 3.5 & 0 & 2.5 & 2.5 & 2.0 \\
\hline 11 & 3.5 & 4.0 & 3.5 & 3.0 & 4.0 & 4.5 & 3.5 & 2.5 & 1.5 & 2.0 & 2.5 & 0 & 1.0 & 2.5 \\
\hline 12 & 3.5 & 5.0 & 3.5 & 4.0 & 4.0 & 4.5 & 3.5 & 3.5 & 2.5 & 3.0 & 2.5 & 1.0 & 0 & 1.5 \\
\hline 13 & 3.0 & 6.5 & 5.0 & 5.5 & 3.5 & 4.0 & 3.0 & 5.0 & 4.0 & 4.5 & 2.0 & 2.5 & 1.5 & 0 \\
\hline
\end{tabular}

Table 2: The freight flow between two depots (one semi-trailer).

\begin{tabular}{lcccccccccccccc}
\hline From & & & & & & & & To & & & & & \\
& $H$ & 1 & 2 & 3 & 4 & 5 & 6 & 7 & 8 & 9 & 10 & 11 & 12 & 13 \\
\hline$H$ & $0^{\mathrm{b}}$ & $1^{\mathrm{a}}$ & 1 & 1 & 1 & 1 & 1 & 1 & 1 & 1 & 1 & 1 & 1 & 1 \\
1 & 1 & 0 & 1 & 0 & 1 & 1 & 0 & 0 & 0 & 0 & 0 & 0 & 0 & 1 \\
2 & 1 & 1 & 0 & 0 & 0 & 0 & 0 & 0 & 1 & 0 & 1 & 1 & 0 & 0 \\
3 & 1 & 0 & 0 & 0 & 1 & 0 & 0 & 0 & 0 & 0 & 1 & 0 & 1 & 1 \\
4 & 1 & 0 & 0 & 1 & 0 & 0 & 0 & 0 & 1 & 1 & 0 & 0 & 0 & 1 \\
5 & 1 & 0 & 0 & 1 & 1 & 0 & 0 & 0 & 0 & 0 & 1 & 0 & 0 & 1 \\
6 & 1 & 0 & 0 & 0 & 1 & 1 & 0 & 0 & 0 & 0 & 1 & 1 & 0 & 0 \\
7 & 1 & 0 & 0 & 0 & 1 & 0 & 0 & 0 & 0 & 0 & 1 & 1 & 1 & 0 \\
8 & 1 & 0 & 1 & 0 & 0 & 0 & 1 & 0 & 0 & 1 & 0 & 1 & 0 & 0 \\
9 & 1 & 0 & 1 & 0 & 1 & 0 & 1 & 0 & 0 & 0 & 0 & 1 & 0 & 0 \\
10 & 1 & 1 & 0 & 0 & 0 & 1 & 0 & 1 & 1 & 0 & 0 & 0 & 0 & 0 \\
11 & 1 & 0 & 1 & 0 & 1 & 0 & 0 & 0 & 1 & 0 & 0 & 0 & 0 & 1 \\
12 & 1 & 0 & 1 & 0 & 0 & 1 & 0 & 0 & 0 & 0 & 0 & 1 & 0 & 1 \\
13 & 1 & 0 & 0 & 0 & 1 & 0 & 1 & 0 & 1 & 1 & 0 & 0 & 0 & 0 \\
\hline
\end{tabular}

a" 1 " denotes that there is one semi-trailer flow between two depots.

b" 0 " denotes that there is no freight flow.

which can generate random arrays from a specified distribution, is used. By RANDOM ("norm",1,1,10,10), a random array is generated. We select the negative positions of the array as nodes and the minimum position as the main depot. The distance between any two nodes is calculated by the gaps of rows and columns. The "RANDOM" function in MATLAB is also used to determine the freight flow between two depots. The network expressed by Table 1 and the flow expressed by Table 2 make up example No. 1. By the above generation method, we produce some transportation networks that are used to test the heuristic algorithm. 
Table 3: The satisfactory solution of the TSRP achieved by the heuristic algorithm.

\begin{tabular}{lcc}
\hline Types of the routes & Form of route & $\begin{array}{c}\text { Working time } \\
\text { needed by } \\
\text { routes (hours) }\end{array}$ \\
\hline $\begin{array}{l}\text { A tractor with two drivers. Two different } \\
\text { customer depots are passed by. }\end{array}$ & 17 \\
The route repeats once a day. & & 17.5 \\
A tractor with two drivers. Three different \\
customer depots are passed by. \\
The route repeats once a day. \\
$\begin{array}{l}\text { A tractor with two drivers. Four different } \\
\text { customer depots are passed by. }\end{array}$ \\
$\begin{array}{l}\text { The route repeats once a day. } \\
\text { A tractor with two drivers. Five different }\end{array}$ \\
$\begin{array}{l}\text { customer depots are passed by. } \\
\text { The route repeats once a day. }\end{array}$ \\
$\begin{array}{l}\text { A } \\
\text { A tractor with two drivers. Six different } \\
\text { customer depots are passed by. }\end{array}$
\end{tabular}

According to some enterprise experience, a driver's on-duty time is 8.5 hours per day. One or two drivers are assigned to a tractor. A tractor with two drivers can work consecutively for no more than 17 hours in a 24-consecutive-hour period. The temporary rest time in customer depots is 0.5 hour, and the residence time in $H$ is 1 hour. By using the approach mentioned in Section 3.1, we attain different types of tractor routes for the No. 1 example. There are 175 elements in the initial solution set. By using the 2-phase approach, we attain the satisfactory solution of the No. 1 example (Table 3). When the enterprise employs tractor routes as the satisfactory solution, it can satisfy 80 percent of all transportation demand. Sixteen tractors and thirty-two drivers are needed during a 24-consecutive-hour period. The total running time of 16 tractors is 230 hours per day. In 15 percent of the total running time, tractors run alone.

It is feasible to propose exact algorithms (e.g., integer programming) for the TSRP when the initial solution set is constructed. We proposed a $0-1$ integer programming for the No. 1 example and attained the exact solution. The exact solution can satisfy 84 percent of all transportation demand. Sixteen tractors and thirty-two drivers are needed during a 24-consecutive-hour period. In 10 percent of the total running time, tractors run alone. We implemented the proposed heuristic algorithm using Matlab and the 0-1 integer programming with QS. Although the exact algorithm can attain a slightly better solution, it requires more calculating time. For the No. 1 example, the solving time using the heuristic algorithm was approximately 80 seconds while that for the exact algorithm was approximately 2000 seconds. We suggest that the heuristic algorithm has an advantage for solving the TSRP. 
Table 4: The results of TSRP experiments achieved by the heuristic algorithm.

\begin{tabular}{|c|c|c|c|c|c|}
\hline \multicolumn{2}{|c|}{ Transportation network } & \multicolumn{3}{|c|}{ On the solution } & \multirow{2}{*}{$\begin{array}{c}\text { Calculation } \\
\text { time } \\
\text { (seconds) }\end{array}$} \\
\hline $\begin{array}{l}\text { Number of } \\
\text { nodes }\end{array}$ & $\begin{array}{l}\text { Number of } \\
\text { freight flows }\end{array}$ & $\begin{array}{l}\text { Fraction of demand } \\
\text { satisfied }(\%)\end{array}$ & $\begin{array}{l}\text { Number of } \\
\text { tractors }\end{array}$ & $\begin{array}{l}\text { Average time of tractors } \\
\text { running alone (hours) }\end{array}$ & \\
\hline 10 & 54 & 65 & 10 & 0.9 & 3 \\
\hline 11 & 60 & 85 & 18 & 1.9 & 5 \\
\hline 12 & 66 & 89 & 17 & 1.8 & 26 \\
\hline 13 & 72 & 92 & 22 & 2.1 & 45 \\
\hline 14 & 78 & 89 & 23 & 1.6 & 78 \\
\hline 15 & 84 & 71 & 18 & 1.8 & 47 \\
\hline 17 & 96 & 89 & 28 & 1.7 & 59 \\
\hline 18 & 102 & 81 & 25 & 1.6 & 86 \\
\hline 19 & 108 & 86 & 32 & 1.7 & 114 \\
\hline 21 & 120 & 74 & 28 & 1.6 & 198 \\
\hline 22 & 126 & 71 & 28 & 1.4 & 234 \\
\hline 23 & 132 & 86 & 37 & 1.8 & 310 \\
\hline
\end{tabular}

We have repeated the generation of random arrays over 50 times to obtain some typical computational networks. The heuristic algorithm was employed on these networks. We ran the experiments of this section on a computer with an AMD Athlon(tm) X2 Dual-Core QL65 running at $2.10 \mathrm{GHz}$ under Windows 7 ultimate (32 bits) with $2 \mathrm{~GB}$ of RAM. Table 4 summarizes the characteristics of each solution in the 12-instance testbed.

\section{Conclusions and Future Work}

In this paper, we proposed a TSRP model and suggested a heuristic algorithm to solve it. The TSRP concentrated on a unit-flow network, and all tractors originated and terminated at a main depot. Unlike most approaches to the TTRP or VRP, the heuristic algorithm for the TSRP did not regard the number of vehicles as a precondition. Therefore, the solution to the TSRP was able to balance the variable costs and service level by altering the vehicle number. The main characteristics of the heuristic algorithm are the initial solution set constructed by the limitation of driver on-duty time and the combination of a two-phase filtration on candidate routes. The computational study shows that our algorithm is feasible and effective for the TSRP. Although some exact algorithms for the TSRP are feasible after the initial solution set is constructed, the heuristic algorithm is efficient because it takes relatively less time to obtain satisfactory solutions. Future research may try to extend the TSRP to include more practical considerations, such as time window constraints. Other efficient heuristics for the TSRP may also be proposed. In this regard, the benchmark instances generated in this study may serve as a testbed for future research to test the efficiency of specific algorithms for TSRP.

\section{Acknowledgments}

This work was partially funded by the Science and Technology Plan of Transportation of Shandong Province (2009R58) and the Fundamental Research Funds for the Central Universities (YWF-10-02-059). This support is gratefully acknowledged. 


\section{References}

[1] S. W. Lin, V. F. Yu, and S. Y. Chou, "Solving the truck and trailer routing problem based on a simulated annealing heuristic," Computers and Operations Research, vol. 36, no. 5, pp. 1683-1692, 2009.

[2] F. Semet and E. Taillard, "Solving real-life vehicle routing problems efficiently using tabu search," Annals of Operations Research, vol. 41, no. 4, pp. 469-488, 1993.

[3] J. C. Gerdessen, "Vehicle routing problem with trailers," European Journal of Operational Research, vol. 93, no. 1, pp. $135-147,1996$.

[4] I. M. Chao, "A tabu search method for the truck and trailer routing problem," Computers and Operations Research, vol. 29, no. 1, pp. 33-51, 2002.

[5] S. Scheuerer, "A tabu search heuristic for the truck and trailer routing problem," Computers and Operations Research, vol. 33, no. 4, pp. 894-909, 2006.

[6] J. Renaud and F. F. Boctor, "A sweep-based algorithm for the fleet size and mix vehicle routing problem," European Journal of Operational Research, vol. 140, no. 3, pp. 618-628, 2002.

[7] H. C. Lau, M. Sim, and K. M. Teo, "Vehicle routing problem with time windows and a limited number of vehicles," European Journal of Operational Research, vol. 148, no. 3, pp. 559-569, 2003.

[8] F. Li, B. Golden, and E. Wasil, "A record-to-record travel algorithm for solving the heterogeneous fleet vehicle routing problem," Computers and Operations Research, vol. 34, no. 9, pp. 2734-2742, 2007.

[9] S. Liu, W. Huang, and H. Ma, "An effective genetic algorithm for the fleet size and mix vehicle routing problems," Transportation Research Part E, vol. 45, no. 3, pp. 434-445, 2009.

[10] E. Cao and M. Lai, "The open vehicle routing problem with fuzzy demands," Expert Systems with Applications, vol. 37, no. 3, pp. 2405-2411, 2010.

[11] M. Caramia and F. Guerriero, "A milk collection problem with incompatibility constraints," Interfaces, vol. 40, no. 2, pp. 130-143, 2010.

[12] D. Pisinger and S. Ropke, "A general heuristic for vehicle routing problems," Computers E Operations Research, vol. 34, no. 8, pp. 2403-2435, 2007.

[13] Y. Marinakis and M. Marinaki, "A hybrid multi-swarm particle swarm optimization algorithm for the probabilistic traveling salesman problem," Computers E Operations Research, vol. 37, no. 3, pp. 432-442, 2010.

[14] A. Garcia-Najera and J. A. Bullinaria, "An improved multi-objective evolutionary algorithm for the vehicle routing problem with time windows," Computers $\mathcal{E}$ Operations Research, vol. 38, no. 1, pp. 287-300, 2011.

[15] L. Hong, "An improved LNS algorithm for real-time vehicle routing problem with time windows," Computers and Operations Research, vol. 39, no. 2, pp. 151-163, 2012.

[16] K. C. Tan, Y. H. Chew, and L. H. Lee, "A hybrid multi-objective evolutionary algorithm for solving truck and trailer vehicle routing problems," European Journal of Operational Research, vol. 172, no. 3, pp. 855-885, 2006.

[17] J. G. Villegas, C. Prins, C. Prodhon, A. L. Medaglia, and N. Velasco, "A GRASP with evolutionary path relinking for the truck and trailer routing problem," Computers and Operations Research, vol. 38, no. 9, pp. 1319-1334, 2011.

[18] J. G. Villegas, C. Prins, C. Prodhon, A. L. Medaglia, and N. Velasco, "GRASP/VND and multi-start evolutionary local search for the single truck and trailer routing problem with satellite depots," Engineering Applications of Artificial Intelligence, vol. 23, pp. 780-794, 2010.

[19] S. W. Lin, V. F. Yu, and S. Y. Chou, "A note on the truck and trailer routing problem," Expert Systems with Applications, vol. 37, no. 1, pp. 899-903, 2010.

[20] S. W. Lin, V. F. Yu, and C. C. Lu, "A simulated annealing heuristic for the truck and trailer routing problem with time windows," Expert Systems with Applications, vol. 38, pp. 15244-15252, 2011.

[21] R. W. Hall and V. C. Sabnani, "Control of vehicle dispatching on a cyclic route serving trucking terminals," Transportation Research Part A, vol. 36, no. 3, pp. 257-276, 2002.

[22] U. Derigs, R. Kurowsky, and U. Vogel, "Solving a real-world vehicle routing problem with multiple use of tractors and trailers and EU-regulations for drivers arising in air cargo road feeder services," European Journal of Operational Research, vol. 213, no. 1, pp. 309-319, 2011.

[23] Y. R. Cheng, B. Liang, and M. H. Zhou, "Optimization for vehicle scheduling in iron and steel works based on semi-trailer swap transport," Journal of Central South University of Technology, vol. 17, no. 4, pp. 873-879, 2010.

[24] B. Liang, Research on semi-trailer loop swap transportation applied in large-scale iron and steel works [M.S. thesis], Central South University, 2009. 


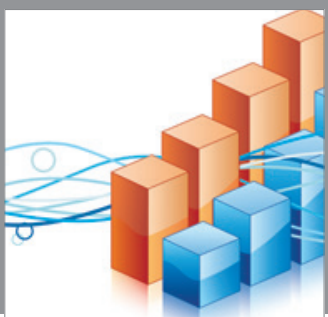

Advances in

Operations Research

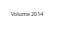

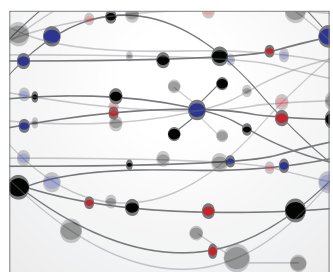

\section{The Scientific} World Journal
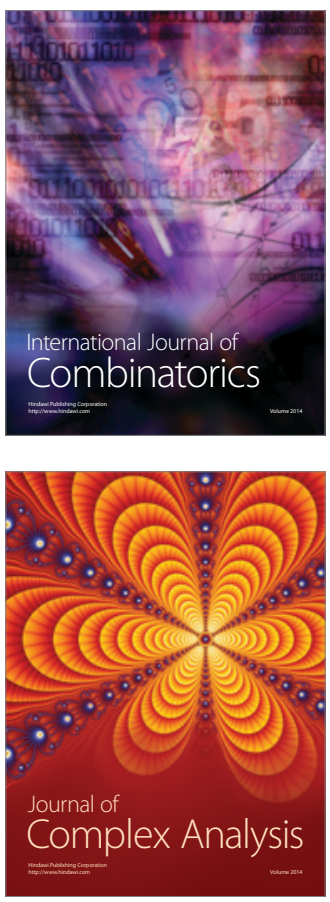

International Journal of

Mathematics and

Mathematical

Sciences
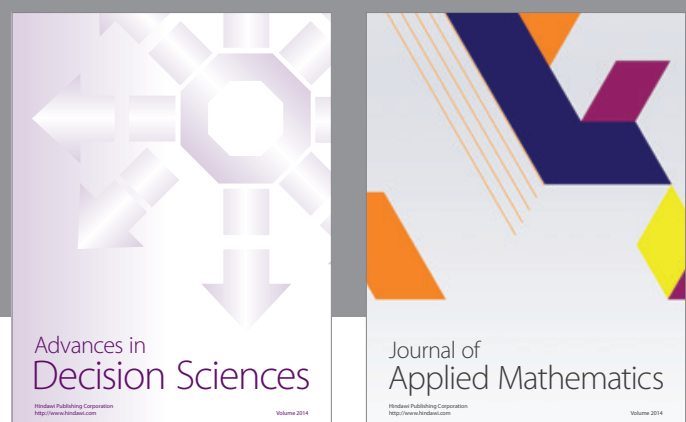

Journal of

Applied Mathematics
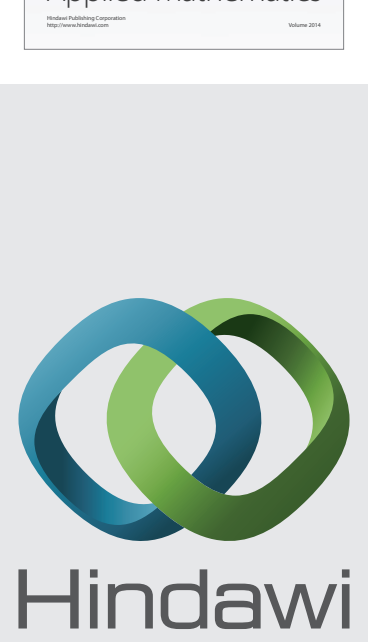

Submit your manuscripts at http://www.hindawi.com
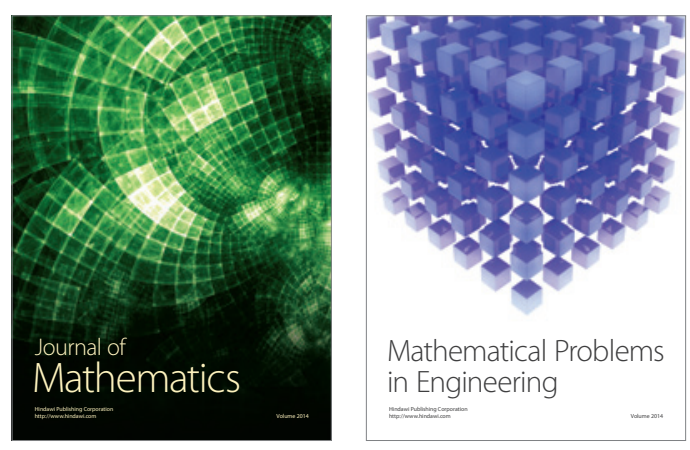

Mathematical Problems in Engineering
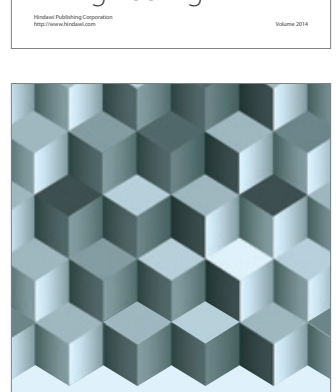

Journal of

Function Spaces
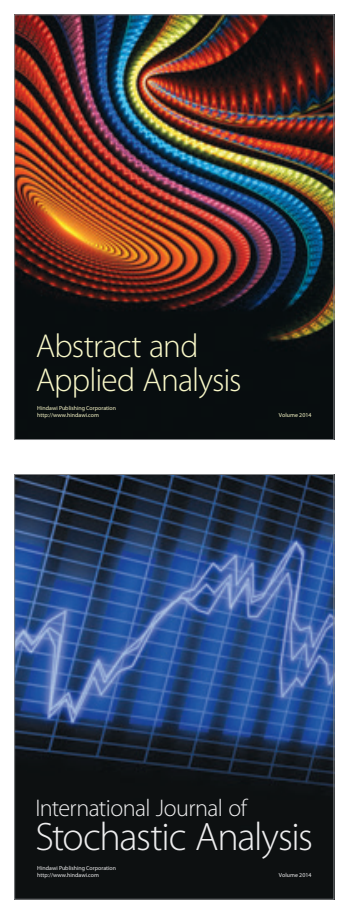

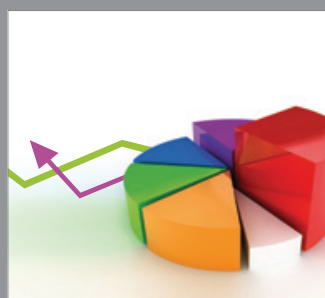

ournal of

Probability and Statistics

Promensencen
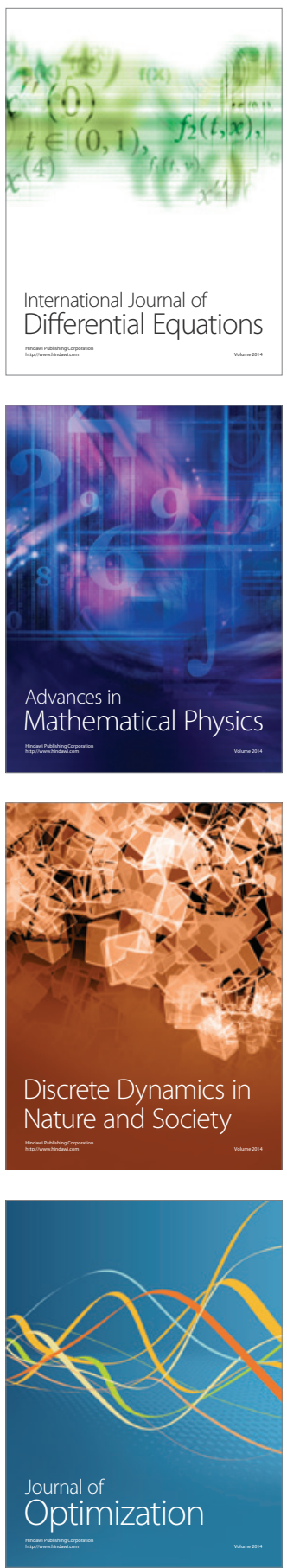